\title{
Leseliste Top 5: Planung und Budgetierung
}

In diesem Teil möchten wir Ihnen regelmäßig fünf besonders lesenswerte Bücher zu einem bestimmten Themengebiet vorstellen:

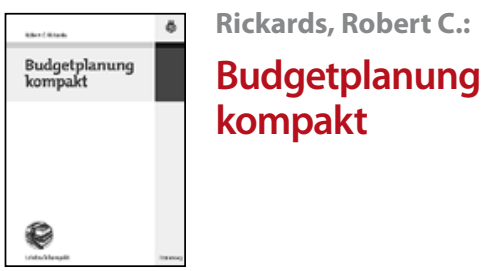

Oldenbourg Verlag, München 2007, 195 S., ISBN: 978-3-486-58385-4, 19,80€

Auf 195 Seiten gibt das Buch von Rickards einen Überblick über die Herkunft des Controllings, den Bereich der Budgetierung sowie dessen neuere Entwicklungen. Im Fokus des Buches steht der traditionelle Budgetierungsprozess. Zudem finden sich kurze Darstellungen weiterer Techniken (z. B. Rollierende Forecasts und Kaizen-Budgets). Besonderheiten sind die enthaltenen Übungen und die Gegenüberstellung der deutschen und englischen Fachtermini. Diese didaktische Ausrichtung und die Praxisorientierung tragen maßgeblich zur Verständlichkeit des Buches bei. Die behandelten Themen lassen sich so leicht erschließen.

Tipp: Dieses Buch richtet sich in erster Linie an Studierende der Betriebswirtschaftslehre. Gleichzeitig ist es auch für Fachfremde und Quereinsteiger leicht verständlich.

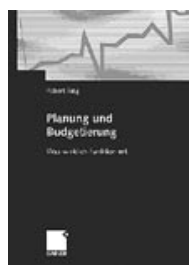

Rieg, Robert:

Planung und Budgetierung -

Was wirklich funktioniert

Gabler Verlag, Wiesbaden 2008, 216 S., ISBN: 978-3-8349-0290-0, 39,90€

Ziel des Buches ist es, Unternehmen bei der Gestaltung und Optimierung ihrer Planungsund Budgetierungsinstrumente zu unterstützen. Nach einigen Grundlagen zur Planung und Budgetierung wird kurz auf Methoden der operativen Planung, speziell Planungs- und Kontrollrechnungen, eingegangen, zudem werden Probleme von Planung und Kontrolle in der Praxis betrachtet.
Der Hauptteil des Buches befasst sich mit der Verbesserung der Planung und Budgetierung, hierbei insbesondere dem Better Budgeting. U. a. werden Verbesserungsmöglichkeiten bei Inhalten, Methoden und Organisation vorgestellt. Darüber hinaus wird das Konzept des Beyond Budgeting präsentiert.

Tipp: Das sehr informative, durch Praxisbeispiele und Abbildungen veranschaulichte Buch richtet sich an die Unternehmenspraxis. Es bietet sowohl Managern als auch Controllern einen guten Einblick in das Thema und Ansatzpunkte für Verbesserungen.

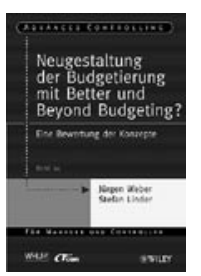

Weber, Jürgen/ Linder, Stefan:

Neugestaltung der Budgetierung mit Better und Beyond Budgeting?

Eine Bewertung der Konzepte

Wiley-VCH Verlag, Weinheim 2008, 99 S., ISBN: 978-3-527-50412-1, 24,90€

Das Buch von Weber/Linder, aus der Reihe Advanced Controlling, stellt die Konzepte der klassischen Budgetierung, des Better/ Advanced Budgeting und des Beyond Budgeting vor. Es beschreibt ihre konzeptionellen Stärken und Schwächen und schildert die Herausforderungen bei der Implementierung. Dabei zeigen die Autoren, dass bei der Planung immer unternehmensindividuelle Lösungen zu erarbeiten sind.

Tipp: Dieses Buch bietet eine kritische Auseinandersetzung mit dem Thema der Budgetierung. Die besprochenen Inhalte richten sich zu großen Teilen an Praktiker, sowohl an Controller als auch an Manager in anderen Unternehmensbereichen.

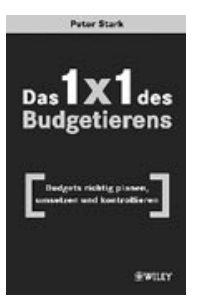

Stark, Peter:

\section{Das 1x1 des} Budgetierens

Budgets richtig planen, umsetzen und kontrollieren

Wiley-VCH Verlag, Weinheim 2006, 198 S., ISBN: 978-3-527-50133-5, 29,90€
Das Buch von Stark soll als Einführung in die Budgetierung dienen. Dazu werden zunächst verschiedene Begrifflichkeiten und der Budgetierungsprozess erläutert sowie rechtliche Rahmenbedingungen (z. B. Basel II, KonTraG) dargelegt. Weiterhin stellt Stark verschiedene Budgetierungssysteme und Kennzahlen vor und geht auf die Anforderungen der Berichtsempfänger sowie Budgetierungssoftware ein. Ein Fallbeispiel und eine Budgetierungscheckliste runden das Buch ab.

Tipp: Das sehr anschauliche und leicht verständliche Buch gibt einen ersten Einblick in das Thema Budgetierung und richtet sich dabei insbesondere an Praktiker.

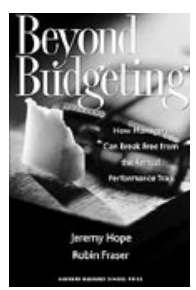

Fraser, Robin/ Hope, Jeremy:

Beyond Budgeting:

How Managers Can Break Free from the Annual

McGraw-Hill Professional, Boston 2003, 336 S., ISBN: 978-1578518661, 29,99€

Die klassische Planung und Budgetierung hindert Unternehmen in ihrer erfolgreichen Entwicklung. Mit dieser provokanten These starten die Autoren und es veranlasst sie, ein alternatives Führungsmodell zu entwerfen - das Beyond Budgeting. Einführend werden einige Nachteile des klassischen Budgetierungsprozesses beschrieben. Diesen Schwächen werden die Möglichkeiten des Beyond Budgeting anhand ausgewählter Beispiele gegenübergestellt. Dabei identifizieren Fraser und Hope zwei wesentliche Gestaltungsfelder: Adaptive Prozesse und Dezentralisierung. Zudem werden einige Instrumente des Controllings (Balanced Scorecard, Activity Based Costing etc.) hinsichtlich ihrer Vereinbarkeit mit dem Beyond Budgeting bewertet.

Tipp: Dieses Buch empfiehlt sich für Praktiker gleichermaßen wie für Wissenschaftler und Studenten.

Fabian Kleinschmit und Julia Schmitz, Vallendar 